\title{
Some panconnected and pancyclic properties of graphs with a local ore-type condition
}

Armen S. Asratian and G. V. Sarkisian

The self-archived postprint version of this journal article is available at Linköping University Institutional Repository (DiVA):

http:/ / urn.kb.se/ resolve?urn=urn:nbn:se:liu:diva-143289

N.B.: When citing this work, cite the original publication.

Asratian, A. S., Sarkisian, G. V., (1996), Some panconnected and pancyclic properties of graphs with a local ore-type condition, Graphs and Combinatorics, 12(3), 209-219.

https:/ / doi.org/ 10.1007/ BF01858455

Original publication available at:

https:// doi.org/ 10.1007/ BF01858455

Copyright:

Publisher URL Missing 


\title{
Some Panconnected and Pancyclic Properties of Graphs with a Local Ore-type Condition
}

\author{
A.S. Asratian ${ }^{1,2}$ and G.V. Sarkisian ${ }^{2}$ \\ 1 Department of Mathematics, University of Umeå, S-901 87 Umeå, Sweden \\ 2 Department of Mathematical Cybernetics, Yerevan State University, Yerevan, 375049, \\ Republic of Armenia
}

\begin{abstract}
Asratian and Khachatrian proved that a connected graph $G$ of order at least 3 is hamiltonian if $d(u)+d(v) \geq|N(u) \cup N(v) \cup N(w)|$ for any path $u w v$ with $u v \notin E(G)$, where $N(x)$ is the neighborhood of a vertex $x$.

We prove that a graph $G$ with this condition, which is not complete bipartite, has the following properties:

a) For each pair of vertices $x, y$ with distance $d(x, y) \geq 3$ and for each integer $n, d(x, y) \leq$ $n \leq|V(G)|-1$, there is an $x-y$ path of length $n$.

(b) For each edge $e$ which does not lie on a triangle and for each $n, 4 \leq n \leq|V(G)|$, there is a cycle of length $n$ containing $e$.

(c) Each vertex of $G$ lies on a cycle of every length from 4 to $|V(G)|$.

This implies that $G$ is vertex pancyclic if and only if each vertex of $G$ lies on a triangle.
\end{abstract}

\section{Introduction}

We use Bondy and Murty [6] for terminology and notation not defined here and consider finite simple graphs only. For each vertex $u$ of a graph $G$ we denote by $N(u)$ the set of all vertices of $G$ adjacent to $u$. The distance between vertices $u$ and $v$ is denoted by $d(u, v)$. A path with $x$ and $y$ as end vertices is called an $x-y$ path. An $x-y$ path is called a Hamilton path if it contains all the vertices of $G$. A graph $G$ is Hamilton-connected if every two vertices of $G$ are connected by a Hamilton path.

Let $G$ be a graph of order $p \geq 3$. $G$ is called panconnected if for each pair of distinct vertices $x$ and $y$ and for each $l, d(x, y) \leq l \leq p-1$, there is an $x-y$ path of length $l$ in $G$. $G$ is called pancyclic if it contains a cycle of length $l$ for each $l$ satisfying $3 \leq l \leq p$. $G$ is called vertex pancyclic (edge pancyclic) if each vertex (edge) of $G$ lies on a cycle of every length from 3 to $p$ inclusive.

Let $M_{1}=\left\{K_{n, n}, n \geq 2\right\}$ and $\mathscr{M}_{2}=\left\{G: K_{n, n} \subseteq G \subseteq K_{n} \vee K_{n}^{c}, n \geq 3\right\}$.

The following results are known.

Theorem 1 (Ore [10]). Let $G$ be a graph of order $p \geq 3$, where $d(u)+d(v) \geq p$ for each pair of nonadjacent vertices $u$ and $v$. Then $G$ is hamiltonian. 
A graph $G$ satisfying the condition of Theorem 1 is called an Ore graph.

Theorem 2 (Bondy [5]). An Ore graph $G$ is pancyclic if. and only if $G \notin \mathscr{M}_{1}$.

Note that Theorem 2 is a corollary from more general theorems of Bondy [5] and Häggkvist, Faudree and Schelp [8].

Theorem $3[7,11]$. Let $G$ be an Ore graph of order $p \geq 4$. Then each vertex of $G$ lies on a cycle of every length from 4 to $p$ inclusive, unless $G \in \mathscr{M}_{1}$.

Theorem 4 (Asratian ${ }^{1}$ and Khachatrian [9]). Let $G$ be a connected graph of order at least 3 where $d(u)+d(v) \geq|N(u) \cup N(v) \cup N(w)|$ for any path $u w v$ with $u v \notin E(G)$. Then $G$ is hamiltonian.

A simpler proof of Theorem 4 was suggested in [2]. Clearly, Theorem 4 implies Theorem 1. Moreover, while Theorem 1 only applies to graphs $G$ with diameter 2 and large edge density $\left(|E(G)| \geq \frac{1}{4} \cdot|V(G)|^{2}\right)$, Theorem 4 applies to infinite classes of graphs $G$ with small edge density $(|E(G)| \leq$ constant $\cdot|V(G)|)$ and large diameter ( $\geq$ constant $\cdot|V(G)|$ ).

Denote by $L_{0}$ the set of graphs of order at least 5 satisfying the conditions of Theorem 4.

Theorem 5 [4]. A graph $G \in L_{0}$ is pancyclic if and only if $G \notin \mathscr{M}_{1}$.

Theorem 6 [1]. A graph $G \in L_{0}$ is Hamilton-connected if and only if it is 3-connected and $G \notin \mathscr{M}_{2}$.

We prove here that a graph $G \in L_{0} \backslash \mathscr{M}_{1}$ has the following properties;

(a) For each pair of vertices $x, y$ with $d(x, y) \geq 3$ and for each integer $n, d(x, y) \leq$ $n \leq|V(G)|-1$, there is an $x-y$ path of length $n$.

(b) For each edge $e$ which does not lie on a triangle and for each $n, 4 \leq n \leq|V(G)|$, there is a cycle of length $n$ containing $e$.

(c) Each vertex of $G$ lies on a cycle of every length from 4 to $|V(G)|$.

The last property implies the following: A graph $G \in L_{0} \backslash \mathscr{M}_{1}$ is vertex pancyclic if and only if each vertex of $G$ lies on a triangle.

Theorems 2,3 and 5 follow from our results.

\section{Notations and Preliminary Results}

Let $P$ be a path of $G$. We denote by $\vec{P}$ the path $P$ with a given orientation and by $\overleftarrow{P}$ the path $P$ with the reverse orientation. If $u, v \in V(P)$, then $u \vec{P} v$ denotes the consecutive vertices of $P$ from $u$ to $v$ in the direction specified by $\vec{P}$. The same vertices, in reverse order, are given by $v \bar{P} u$. We use $w^{+}$to denote the successor of $w$

1 In [9] the last name of the first author was transcribed as Hasratian. 
on $\vec{P}$ and $w^{-}$to denote its predecessor. Also we denote by $N(P)$ the set of vertices $v$ outside $P$ with $N(v) \cap V(P) \neq \varnothing$. If $W \subseteq V(P)$ then $W^{+}=\left\{w^{+} / w \in W\right\}$ and $W^{-}=\left\{w^{-} / w \in W\right\}$. We will say that a path $\vec{P}$ contains a triangle $a_{1} a_{2} a_{3} a_{1}$ if $a_{1}, a_{2}, a_{3} \in V(P), a_{1} a_{3} \in E(G)$ and $a_{1}^{+}=a_{2}=a_{3}^{-}$. A path $\vec{P}$ containing a triangle $\Delta$ is denoted by $\vec{P}^{A}$. The set of all triangles contained in $\vec{P}^{A}$ we denote by $T\left(\vec{P}^{A}\right)$.

Analogous terminology is used with respect to cycles as well.

We assume that an $x-y$ path $\vec{P}$ has an orientation from $x$ to $y$.

A path (cycle) on $n$ vertices will be denoted by $P_{n}$ (respectively, $C_{n}$ ).

Let $A$ and $B$ be two disjoint subsets of vertices of a graph $G$. We denote by $\varepsilon(A, B)$ the number of edges in $G$ with one end in $A$ and the other in $B$.

Proposition 1 [9]. $G \in L_{0}$ if and only if for any path $u w v$ with $u v \notin E(G)|N(u) \cap N(v)| \geq$ $|N(w) \backslash(N(u) \cup N(v))|$ holds.

Corollary 1. If $G \in L_{0}$ then $G$ is 2-connected and $|N(u) \cap N(v)| \geq 2$ for each pair of vertices $u, v$ with $d(u, v)=2$.

Proof. Let $d(u, v)=2$ and $w \in N(u) \cap N(v)$. Then $u, v \in N(w) \backslash(N(u) \cup N(v))$. Therefore, by Proposition 1, $|N(u) \cap N(v)| \geq 2$. This implies 2-connectedness of $G$.

Proposition 2. Let $G \in L_{0}$ and $x, y$ be two distinct vertices of $G$ with $d(x, y)=l \geq 3$. Then there exists an $x-y$ path $P_{l+2}^{4}$.

Proof. Let $P_{l+1}=u_{0} u_{1} \ldots u_{l}$ be an $x-y$ path of length $l$ where $u_{0}=x$ and $u_{l}=y$. Since $P_{l+1}$ is an $x-y$ path of minimum length then

$$
N\left(u_{i}\right) \cap V\left(P_{l+1}\right)=\left\{u_{i-1}, u_{i+1}\right\} \quad \text { for each } i=1, \ldots, l-1 .
$$

Suppose that no vertex $v$ outside $P_{l+1}$ is adjacent to two consecutive vertices of $P_{l+1}$. Then, since $u_{1} \in N\left(u_{0}\right) \cap N\left(u_{2}\right)$ and $u_{2} \in N\left(u_{1}\right) \cap N\left(u_{3}\right)$ we obtain from (1), using Proposition 1, the following:

$$
\begin{aligned}
& d\left(u_{2}\right)-1 \geq\left|N\left(u_{0}\right) \cap N\left(u_{2}\right)\right| \geq\left|N\left(u_{1}\right) \backslash\left(N\left(u_{0}\right) \cup N\left(u_{2}\right)\right)\right|=d\left(u_{1}\right), \\
& d\left(u_{1}\right)-1 \geq\left|N\left(u_{1}\right) \cap N\left(u_{3}\right)\right| \geq\left|N\left(u_{2}\right) \backslash\left(N\left(u_{1}\right) \cup N\left(u_{3}\right)\right)\right|=d\left(u_{2}\right) .
\end{aligned}
$$

But (2) contradicts (3). Hence there are vertices $u_{i}$ and $v$ such that $0 \leq i \leq l-1$, $v \notin P_{l+1}$ and $v u_{i}, v u_{i+1} \in E(G)$. This implies that there is an $x-y$ path $P_{l+2}^{A}=$ $u_{0} \ldots u_{i} v u_{1+i} \ldots u_{l}$ of length $l+1$ with $\Delta=u_{i} v u_{i+1} u_{i}$.

Proposition 3. Let $G \in L_{0} \backslash \mathscr{M}_{1}$. Then each edge of $G$ lies on a triangle or on a cycle $C_{5}^{A}$.

Proof. Let $G \in L_{0}$ and assume that there is an edge $e$ of $G$ which lies neither on a triangle nor on a cycle $C_{5}^{A}$. Let $e=v_{1} w_{1}, d\left(w_{1}\right) \geq d\left(v_{1}\right)$ and $N\left(w_{1}\right)=\left\{v_{1}, \ldots, v_{n}\right\}$. Clearly, $v_{1} v_{j} \notin E(G)$ for each $j=2, \ldots, n$ because $e$ does not lie on a triangle. Since $d\left(v_{1}, v_{2}\right)=2$ there is a vertex $w_{2} \in N\left(v_{1}\right) \cap N\left(v_{2}\right), w_{2} \neq w_{1}$. Clearly, $v_{2} v_{j} \notin E(G)$ for each $j, 3 \leq j \leq n$. (Otherwise $e$ lies on a cycle $C_{5}^{A}$.) Then, using Proposition 1, we have

$$
n=d\left(w_{1}\right) \geq d\left(v_{1}\right) \geq\left|N\left(v_{1}\right) \cap N\left(v_{2}\right)\right| \geq\left|N\left(w_{1}\right) \backslash\left(N\left(v_{1}\right) \cup N\left(v_{2}\right)\right)\right|=\left|N\left(w_{1}\right)\right|=n .
$$


Therefore $n=d\left(v_{1}\right)=\left|N\left(v_{1}\right) \cap N\left(v_{2}\right)\right|$. Let $N\left(v_{1}\right) \cap N\left(v_{2}\right)=\left\{w_{1}, \ldots, w_{n}\right\}$. Clearly, $w_{i} w_{j} \notin E(G)$ for each pair $i, j$ where $2 \leq i<j \leq n$. (Otherwise there is a cycle $C_{5}^{4}$ containing e.) Since $d\left(w_{1}, w_{i}\right)=2$ then, using Proposition 1, we have $\left|N\left(w_{1}\right) \cap N\left(w_{i}\right)\right| \geq\left|N\left(v_{1}\right) \backslash\left(N\left(w_{i}\right) \cup N\left(w_{1}\right)\right)\right|=\left|N\left(v_{1}\right)\right|=n$ for each $i=2, \ldots, n$. Therefore $w_{i} v_{j} \in E(G)$ for each pair $i, j$, where $1 \leq i, j \leq n$.

- Let us show that $V(G)=\left\{v_{1}, \ldots, v_{n}, w_{1}, \ldots, w_{n}\right\}$. Suppose that there is a vertex $u \in V(G) \backslash\left\{v_{1}, \ldots, v_{n}, w_{1}, \ldots, w_{n}\right\}$ such that $u w_{i} \in E(G)$ for some $i, 2 \leq i \leq n$. Then $u v_{j} \notin E(G)$ for each $j=2, \ldots, n$. (Otherwise there is a cycle $C_{5}^{A}$ containing $e$.) Since $d\left(u, v_{1}\right)=2$ then, using Proposition 1, we have

$$
\left|N(u) \cap N\left(v_{1}\right)\right| \geq\left|N\left(w_{i}\right) \backslash\left(N(u) \cup N\left(v_{1}\right)\right)\right| \geq n+1
$$

because $u, v_{1}, \ldots, v_{n} \in N\left(w_{i}\right) \backslash\left(N(u) \cup N\left(v_{1}\right)\right)$. But then $n=d\left(v_{1}\right) \geq\left|N\left(v_{1}\right) \cap N(u)\right|$, a contradiction. Hence $N\left(w_{i}\right)=\left\{v_{1}, \ldots, v_{n}\right\}$ for each $i=1, \ldots, n$.

Using the same arguments it is possible to prove that $N\left(v_{j}\right)=\left\{w_{1}, \ldots, w_{n}\right\}$ for each $j=1, \ldots, n$. Consequently $G=K_{n, n}$.

Corollary 2. Let $G \in L_{0} \backslash \mathscr{M}_{1}$. Then each vertex of $G$ lies on a triangle or on a cycle $C_{5}^{4}$.

Corollary 3. Each graph $G \in L_{0} \backslash \mathscr{M}_{1}$ contains a triangle.

\section{Main Results}

Proposition 4. Let $G \in L_{0}$ and $x, y$ be two distinct vertices of $G$. If there is an $x-y$ path $P_{n}$ such that $n=|V(G)|-1$ and $|N(v) \cap\{x, y\}| \leq 1$ for the unique vertex $v$ outside $P_{n}$ then there exists a Hamilton $x-y$ path of $G$.

Proof. W.l.o.g. we assume $v y \notin E(G)$. Let $W_{v}$ denote the set $N(v) \cap V\left(P_{n}\right)$ and $p=\left|W_{v}\right|$. If $v w^{+} \in E(G)$ for some $w \in W_{v}$ then there is a Hamilton $x-y$ path. Suppose that $v w^{+} \notin E(G)$ for each $w \in W_{v}$. Since $n=|V(G)|-1$ we have that $N(v) \cap N\left(w^{+}\right) \subseteq W_{v}$ for each $w \in W_{v}$. Suppose that $w^{+} g^{+} \notin E(G)$ for each pair $w$, $g \in W_{v}$. Then

$$
\sum_{w \in W_{v}}\left|N(v) \cap N\left(w^{+}\right)\right| \leq \varepsilon\left(W_{v}, W_{v}^{+}\right)
$$

and

$$
\sum_{w \in W_{v}}\left|N(w) \backslash\left(N(v) \cup N\left(w^{+}\right)\right)\right| \geq \varepsilon\left(W_{v}, W_{v}^{+}\right)+p
$$

since $v \in N(w) \backslash\left(N(v) \cup N\left(w^{+}\right)\right)$for each $w \in W_{v}$. By Proposition 1 we have

$$
\sum_{w \in W_{v}}\left|N(v) \cap N\left(w^{+}\right)\right| \geq \sum_{w \in W_{v}}\left|N(w) \backslash\left(N(v) \cap N\left(w^{+}\right)\right)\right| .
$$

But the last inequality contradicts the previous two. So $w^{+} g^{+} \in E(G)$ for some pair of vertices $w, g$ where $w$ occur on $P_{n}$ before $g$. Then there exists a Hamilton $x-y$ path $P_{n+1}=x \vec{P}_{n} w v g \vec{P}_{n} w^{+} g^{+} \vec{P}_{n} y$. 
The next technical lemma plays a key role in the proofs of the subsequent theorems.

Lemma. Let $G \in L_{0}$ and $x, y$ be two distinct vertices of $G$. If there exists an $x-y$ path $P_{n}^{\Delta}$ such that $4 \leq n \leq|V(G)|-2$ and $|N(v) \cap\{x, y\}| \leq 1$ for each $v \in V(G) \backslash V\left(P_{n}^{d}\right)$ then there exists an $x-y$ path $P_{n+t}^{A_{1}}$ such that $1 \leq t \leq 2$ and $V\left(P_{n}^{\Delta}\right) \subset V\left(P_{n+t}^{\Delta_{1}}\right)$.

The proof of the lemma will be given later.

Theorem 7. Let $G \in L_{0}$ and $e=x y$ be an edge of $G$ which does not lie on a triangle. Then e lies on a cycle $C_{n}$ for each $n, 4 \leq n \leq|V(G)|$.

Proof. It is sufficient to prove that there exists an $x-y$ path $P_{n}$ for each $n$, $4 \leq n \leq|V(G)|$. Since $e$ does not lie on a triangle then, by Proposition 3, $e$ lies on a cycle $C_{5}^{\Delta}$. Hence there is an $x-y$ path $P_{4}$ and an $x-y$ path $P_{5}^{d}$. Suppose that there exist $x-y$ paths $P_{4}, \ldots, P_{n-1}$ and an $x-y$ path $P_{n}^{\Delta}$ for some $n, 5 \leq n \leq|V(G)|-1$. If $n=|V(G)|-1$ then, by Proposition 4 , there exists a Hamilton $x-y$ path. If $n \leq|V(G)|-2$ then, by the lemma, there exists an $x-y$ path $P_{n+t}^{\Delta_{2}}$ where $1 \leq t \leq 2$. If $t=2$ and $A_{1}=w^{-} w w^{+} w^{-}$then we can obtain an $x-y$ path $P_{n+1}$ by deleting the vertex $w$ from $P_{n+2}^{d_{1}}$.

Repetition of our argument shows that there is an $x-y$ path $P_{n}$ for each $n$, $4 \leq n \leq|V(G)|$.

Using Proposition 2 instead of Proposition 3 and the same arguments as in the proof of Theorem 7 , we can prove the following.

Theorem 8. Let $G \in L_{0}$ and $x, y$ be two distinct vertices of $G$ with $d(x, y) \geq 3$. Then for each $n, d(x, y)+1 \leq n \leq|V(G)|$, there exists an $x-y$ path $P_{n}$.

Let a cycle $\vec{C}$ contain a triangle $\Delta=a_{1} a_{2} a_{3} a_{1}$. We call the vertex $a_{2}$ a centre of $\Delta$ and denote it by $s(\Delta)$.

Proposition 5. Let $G \in L_{0} \backslash \mathscr{M}_{1}$. Then each vertex $g$ of $G$ lies on a cycle $C_{4}^{4}$ or on a cycle $C_{s}^{d}$ such that $g \neq s(\Delta)$.

Proof. If $g$ does not lie on a triangle then, by Corollary $2, g$ lies on a cycle $C_{5}^{A}$ and $g \neq s(\Delta)$. Now suppose that $g$ lies on a triangle $x g y x$.

Case 1. $d(g)=2$. Since $|V(G)| \geq 5$ and, by Corollary $1, G$ is 2 -connected then must exist a vertex $v$ with $d(g, v)=2$. Clearly, $|N(g) \cap N(v)| \geq 2$. Since $d(g)=2$ then $N(g) \cap N(v)=\{x, y\}$. So we have a cycle $C_{4}^{\Delta}=x v y g x$ with $\Delta=x v y x$ and $s(\Delta)=v$.

Case 2. $d(g) \geq 3$ and $g$ has a common neighbour with $x$ or $y$ outside $\Delta$.

W.l.o.g. suppose $x v, v g \in E(G)$ for some $v \notin\{x, y, g\}$. Then there is a cycle $C_{4}^{\Delta}=x v g y x$ with $\Delta=x v g x$ and $s(\Delta)=v$.

Case $3 . d(g) \geq 3$ and $g$ has no common neighbour with $x$ and $y$ outside $A$.

Consider a vertex $v \in N(g) \backslash\{x, y\}$. Since $d(v, x)=2$ we have that $|N(x) \cap N(v)| \geq 2$ and there exists a vertex $z \in N(v) \cap N(x), z \neq g$. Now we have a cycle $C_{5}^{A}=x y g v z x$ with $\Delta=x y g x$ and $s(\Delta)=y$. 
Theorem 9. Let $G \in L_{0} \backslash \mathscr{M}_{1}$. Then for each vertex $g$ of $G$ and for each $n, 4 \leq n \leq$ $|V(G)|$, there is a cycle of length $n$ containing $g$.

Proof. By Proposition 5, $g$ lies on a cycle $C_{r}^{A}$ where $s(\Delta) \neq g$ and $4 \leq r \leq 5$. Hence there is a cycle $C_{4}$ containing $g$.

Suppose there exist cycles $C_{4}, \ldots, C_{n}, 4 \leq n \leq|V(G)|-1$, containing $g$ such that the last cycle $C_{n}$ contains a triangle $\Delta=a_{1} a_{2} a_{3} a_{1}$ with $a_{2}=s(\Delta) \neq g$ i.e. $C_{n}=C_{n}^{\Delta}$. If there exists a vertex $v \in N\left(C_{n}^{\Delta}\right)$ which is adjacent to two consecutive vertices $w$ and $w^{+}$of $C_{n}^{\Delta}$ then there is a cycle $C_{n+1}^{\Delta_{1}}=w v w^{+} \vec{C}_{n}^{\Delta} w$ with $\Delta_{1}=w v w^{+} w$ where $s\left(\Delta_{1}\right)=v \neq g$. Now let no vertex $v \in N\left(C_{n}^{\Delta}\right)$ be adjacent to two consecutive vertices of $C_{n}^{A}$. Consider a vertex $v \in N\left(C_{n}^{A}\right)$ and the triangle $\Delta=a_{1} a_{2} a_{3} a_{1}$. Clearly, $g \neq a_{2}$.

Let us define vertices $x$ and $y$, a triangle $\Delta_{0}$ and an $x-y$ path $P_{n}^{S_{0}}$ in the following way.

If $g \in W_{v}$ and $g \neq a_{3}$ then $x=g, y=g^{-}, \Delta_{0}=\Delta$ and $P_{n}^{\Delta_{0}}=g \vec{C}_{n}^{\Delta} g^{-}$.

If $g \in W_{v}$ and $g=a_{3}$, then $x=g, y=g^{+}, \Delta_{0}=a_{3} a_{2} a_{1} a_{3}$ and $P_{n}^{\Delta_{0}}=g \bar{C}_{n}^{\Delta} g^{+}$.

If $g \notin W_{v}$ and $g \neq a_{1}$ then $x=g^{+}, y=g, \Delta_{0}=\Delta$ and $P_{n}^{\Delta_{0}}=g^{+} \vec{C}_{n}^{\Delta} g$.

If $g \notin W_{v}$ and $g=a_{1}$ then $x=g^{-}, y=g, \Delta_{0}=a_{3} a_{2} a_{1} a_{3}$ and $P_{n}^{d_{0}}=g^{-} \bar{C}_{n}^{\Delta} g$.

Clearly, $|N(v) \cap\{x, y\}| \leq 1$ for each $v \in N\left(P_{n}^{\Delta_{0}}\right)$. If $n=|V(G)|-1$ then, by Proposition 4, there exists an Hamilton $x-y$ path. Clearly, since $x$ and $y$ are adjacent, there also exists a Hamilton cycle of $G$. If $n \leq|V(G)|-2$ then, by the lemma, there is an $x-y$ path $P_{n+t}^{A_{1}}$ such that $1 \leq t \leq 2$ and $V\left(P_{n}^{A_{0}}\right) \subset V\left(P_{n+t}^{A_{1}}\right)$. Since $g \in\{x, y\}$ then $g \neq s\left(\Delta_{1}\right)$. The path $P_{n+t}^{\Delta_{1}}$ define a cycle $C_{n+t}^{\Delta_{1}}$. If $t=2$ we can obtain a cycle $C_{n+1}$ containing $g$ by deleting the centre $s\left(\Delta_{1}\right)$ from $C_{n+2}^{A_{1}}$.

Repetition of our argument shows that there is a cycle $C_{n}$ for each $n, 4 \leq n \leq$ $|V(G)|$.

Clearly, Theorem 5 follows from Theorem 9 and Corollary 3. Using Theorem 9 we can formulate a criterion for a graph $G \in L_{0}$ to be vertex pancyclic.

Theorem 10. A graph $G \in L_{0}$ is vertex pancyclic if and only if every vertex of $G$ lies on a triangle.

Corollary 5. Let $G$ be a connected graph of order at least 3 where $d(u)+d(v) \geq$ $\mid N(u) \cup N(v) \cup N(w)\}+1$ for any path uwv with $u v \notin E(G)$. Then $G$ is vertex pancyclic if and only if every vertex of $G$ lies on a triangle.

Let us point out that for each $p \geq 7$ the graph $K_{3}^{c} \vee\left(K_{1}+K_{p-4}\right)$ satisfies the condition of Corollary 5 but contains a vertex that does not lie on a triangle. The next result immediately follows from Theorem 9 .

Corollary 6. Let $G \in L_{0} \backslash \mathscr{M}_{1}$ be a graph of order $p \geq 5$ where $d(u)+d(v) \geq p$ for each pair of vertices $u, v$ with $d(u, v)=2$. Then each vertex of $G$ lies on a cycle of every length from 4 to $p$.

Finally we give the proof of the lemma.

Proof. Since $G$ is connected and $n<|V(G)|$ then $N\left(P_{n}^{\Delta}\right) \neq \varnothing$. For each $v \in N\left(P_{n}^{A}\right)$ 
we denote by $W_{v}$ the set $N(v) \cap V\left(P_{n}^{\Delta}\right)$. Let $U_{1}=\left\{v \in N\left(P_{n}^{\Delta}\right) /\left|W_{v}\right|=1\right\}$ and $U_{2}=$ $N\left(P_{n}^{d}\right) \backslash U_{1}$.

Suppose that there does not exist any $x-y$ path $P_{t+n}^{A_{1}}$ such that $1 \leq t \leq 2$ and $V\left(P_{n}^{d}\right) \subset V\left(P_{n+\mathrm{t}}^{A_{1}}\right)$. Then the following properties hold.

Property 1. $v w^{+} \notin E(G)$ for each $v \in N\left(P_{n}^{\Delta}\right)$ and each $w \in W_{v}$.

Property 2. If $v \in U_{1}, W_{v}=\{w\}$ and $w \notin\{x, y\}$ then the set $T\left(\vec{P}_{n}^{\Delta}\right)$ contains the unique triangle $w^{-} w w^{+} w^{-}$.

Proof. Let $a_{1} a_{2} a_{3} a_{1}$ be a triangle from the set $T\left(\vec{P}_{n}^{\Delta}\right)$. Suppose $a_{2} \neq w$. Since $d\left(v, w^{-}\right)=2=d\left(v, w^{+}\right)$then, by Corollary 1 , there exist vertices $v_{1}$ and $v_{2}$ such that $v_{1} \in\left(N(v) \cap N\left(w^{-}\right)\right) \backslash V\left(P_{n}^{\Delta}\right)$ and $v_{2} \in\left(N(v) \cap N\left(w^{+}\right)\right) \backslash V\left(P_{n}^{\Delta}\right)$. This gives an $x-y$ path

$$
P_{n+2}^{A_{1}}= \begin{cases}x \vec{P}_{n}^{A} w^{-} v_{1} v w \vec{P}_{n}^{A} y & \text { if } a_{2} \in w^{+} \vec{P}_{n}^{A} y \\ x \vec{P}_{n}^{A} w v v_{2} w^{+} \vec{P}_{n}^{A} y & \text { if } a_{2} \in x \vec{P}_{n}^{A} w^{-}\end{cases}
$$

with $\Delta_{1}=a_{1} a_{2} a_{3} a_{1}$ such that $V\left(P_{n}^{S}\right) \subset V\left(P_{n+2}^{\Delta_{1}}\right)$, a contradiction.

Property 3. $U_{2} \neq \varnothing$.

Proof. Suppose that $U_{2}=\varnothing$. Let $v \in U_{1}, W_{v}=\left\{w_{1}\right\}$ and $\Delta_{1}=a_{1} a_{2} a_{3} a_{1}$ be a triangle from the set $T\left(\vec{P}_{n}^{\Delta}\right)$. Three cases are possible: $w_{1}=x, w_{1}=y$ and $w_{1} \notin\{x, y\}$.

Let $w_{1}=x$. Since $d\left(v, w_{1}^{+}\right)=2$ and $\left|W_{v}\right|=1$ then, by Corollary 1 , there is a vertex $v_{1} \in\left(N(v) \cap N\left(w_{1}^{+}\right)\right) \backslash V\left(P_{n}^{d}\right)$. Hence, by Property $2, a_{1}=w_{1}, a_{2}=w_{1}^{+}$and $a_{1} a_{2} a_{3} a_{1}$ is the unique triangle in the set $T\left(\vec{P}_{n}^{d}\right)$. Since $v_{1} \in U_{1}, d\left(v_{1}, a_{3}\right)=2$ and $\left|W_{v_{1}}\right|=1$ then, by Corollary 1 , there is a vertex $v_{2} \in\left(N\left(v_{1}\right) \cap N\left(a_{3}\right)\right) \backslash V\left(P_{n}^{\Delta}\right)$. Clearly, $v_{2} \in U_{1}, W_{v_{2}}=\left\{a_{3}\right\}$ and $a_{2}^{-} a_{2}^{+} \notin E(G)$. This contradicts Property 2.

Using the same arguments we obtain contradictions in the cases $w=y$ and $w \notin\{x, y\}$. Hence, $U_{2} \neq \varnothing$.

Property 4. Let $v \in U_{2}, v y \notin E(G)$ and $Q \subseteq W_{v}=\left\{w_{1}, \ldots, w_{p}\right\}$. Then

$$
\sum_{w_{i} \in Q}\left|N(v) \cap N\left(w_{i}^{+}\right)\right| \geq \sum_{w_{i} \in Q}\left|N\left(w_{i}\right) \backslash\left(N(v) \cup N\left(w_{i}^{+}\right)\right)\right|
$$

Furthermore, if $a_{1} a_{2} a_{3} a_{1}$ is a triangle from $T\left(\vec{P}_{n}^{d}\right)$ with $\left\{a_{1}, a_{2}\right\} \cap Q=\varnothing$ then

$$
N(v) \cap N\left(w_{i}^{+}\right) \subseteq W_{v} \quad \text { for each } w_{i} \in Q
$$

and

$$
w_{i}^{+} w_{j}^{+} \notin E(G) \quad \text { for each pair of vertices } w_{i}, w_{j} \in Q
$$

Proof. Since $d\left(v, w_{i}^{+}\right)=2$ for each $w_{i} \in Q$ then (4) follows from Proposition 1. If (5) does not hold then there exist a vertex $v_{1} \in\left(N(v) \cap N\left(w_{i}^{+}\right)\right) \backslash W_{v}$ for some $w_{i} \in Q$ and an $x-y$ path $P_{n+2}^{A_{t}}=x \vec{P}_{n}^{A} w_{i} v v_{1} w_{i}^{+} \vec{P}_{n}^{A} y$ with $\Delta_{1}=a_{1} a_{2} a_{3} a_{1}$, a contradiction. So, (5) must hold.

If $w_{i}^{+} w_{j}^{+} \in E(G)$ for some pair $w_{i}, w_{j} \in Q, i<j$, then there is an $x-y$ path $P_{n+1}^{A_{1}}=x \vec{P}_{n}^{\Delta} w_{i} v w_{j} \overleftarrow{P}_{n}^{\Delta} w_{i}^{+} w_{j}^{+} \vec{P}_{n}^{\Delta} y$ with 


$$
\Delta_{1}= \begin{cases}a_{1} a_{2} a_{3} a_{1} & \text { if } a_{1} \notin w_{i}^{+} \vec{P}_{n}^{\Delta} w_{j} \\ a_{3} a_{2} a_{1} a_{3} & \text { otherwise }\end{cases}
$$

a contradiction. So, (6) holds.

Property 5. Let $v \in U_{2}$ and $v y \notin E(G)$. If $a_{1} a_{2} a_{3} a_{1}$ is a triangle from the set $T\left(\vec{P}_{n}^{\Delta}\right)$ then $W_{v} \cap\left\{a_{1}, a_{2}\right\} \neq \varnothing$.

Proof. Let $w_{1}, \ldots, w_{p}$ denote the vertices of $W_{v}$ occurring on $\vec{P}_{n}^{A}$ in the order of their indices. Suppose $\left\{a_{1}, a_{2}\right\} \cap W_{v}=\varnothing$. Set $Q=W_{v}$. Then, by Property 4, we have (4), (5) and (6). Furthermore, we have

$$
\sum_{w_{i} \in Q}\left|N(v) \cap N\left(w_{i}^{+}\right)\right| \leq \varepsilon\left(Q, Q^{+}\right)
$$

and

$$
\sum_{w_{i} \in Q}\left|N\left(w_{i}\right) \backslash\left(N(v) \cup N\left(w_{i}^{+}\right)\right)\right| \geq \varepsilon\left(Q, Q^{+}\right)+p
$$

because $v \notin Q^{+}$and $v \in N\left(w_{i}\right) \backslash\left(N(v) \cup N\left(w_{i}^{+}\right)\right)$for each $i=1, \ldots, p$. But (7) and (8) contradict (4). So, $\left\{a_{1}, a_{2}\right\} \cap W_{v} \neq \varnothing$.

Property 6. $\left|W_{v}\right|=2$ for each vertex $v \in U_{2}$. Moreover $w^{-} w^{+} \in E(G)$ for each $w \in W_{v} \backslash\{x, y\}$.

Proof. Let $v \in U_{2}$. Then $\left|W_{v} \cap\{x, y\}\right| \leq 1$ by the assumption (in the lemma). W.l.o.g. we may assume that $v y \notin E(G)$. Let $w_{1}, \ldots, w_{p}$ be the vertices of $W_{v}$ occurring on $\vec{P}_{n}^{\Delta}$ in the order of their indices. By Property 5 we have that $W_{v} \cap\left\{a_{1}, a_{2}\right\} \neq \varnothing$ for each triangle $a_{1} a_{2} a_{3} a_{1}$ from the set $T\left(\vec{P}_{n}^{d}\right)$. Let $k$ be the minimum $i, 1 \leq i \leq p$, for which $w_{i}^{-} w_{i}^{+} \in E(G)$ or $w_{i} w_{i}^{++} \in E(G)$.

Case 1. $k<p$.

Let $a_{1} a_{2} a_{3} a_{1}$ be a triangle with $w_{k} \in\left\{a_{1}, a_{2}\right\}$. Set $Q=W_{v} \backslash\left\{w_{k}\right\}$. Since $Q \cap\left\{a_{1}, a_{2}\right\}=\varnothing$ then by Property 4 all of (4), (5) and (6) hold. Since the vertices $w_{k}$ and $w_{i}^{+}$can be adjacent for each $w_{i} \in Q$, we have

$$
\sum_{w_{i} \in Q}\left|N(v) \cap N\left(w_{i}^{+}\right)\right| \leq \varepsilon\left(Q, Q^{+}\right)+p-1
$$

If $w_{k+1}^{-} w_{k+1}^{+} \notin E(G)$ then

$$
\sum_{w_{i} \in Q}\left|N\left(w_{i}\right) \backslash\left(N(v) \cup N\left(w_{i}^{+}\right)\right)\right| \geq \varepsilon\left(Q, Q^{+}\right)+p
$$

because $w_{k+1}^{-} \notin Q^{+}, \quad w_{k+1}^{-} \in N\left(w_{k+1}\right) \backslash\left(N(v) \cup N\left(w_{k+1}^{+}\right)\right)$and $v \notin Q^{+}, \quad v \in N\left(w_{i}\right) \backslash$ $\left(N(v) \cup N\left(w_{i}^{+}\right)\right)$for each $w_{i} \in Q$.

But (9) and (10) contradict (4). Consequently $w_{k+1}^{-} w_{k+1}^{+} \in E(G)$. Moreover note that $w_{k}^{+} \neq w_{k+1}^{-}$, since otherwise we find an $x-y$ path of length $n+1$ containing a triangle. Now set $Q=W_{v} \backslash\left\{w_{k+1}\right\}$. Then, by Property 4, all of (4), (5) and (6) also hold for the new $Q$. Clearly, $w_{j}^{+} w_{k+1} \notin E(G)$ for each $j \neq k, 1 \leq j \leq p$. (Otherwise there is an $x-y$ path $P_{n+1}^{d_{1}}$ with $\Delta_{1}=a_{1} a_{2} a_{3} a_{1}$, a contradiction.) Hence, we have

$$
\sum_{w_{i} \in Q}\left|N(v) \cap N\left(w_{i}^{+}\right)\right| \leq \varepsilon\left(Q, Q^{+}\right)+1 \text {. }
$$


Furthermore,

$$
\sum_{w_{i} \in Q}\left|N\left(w_{i}\right) \backslash\left(N(v) \cup N\left(w_{i}^{+}\right)\right)\right| \geq \varepsilon\left(Q, Q^{+}\right)+p-1
$$

because $v \notin Q^{+}$and $v \in N\left(w_{i}\right) \backslash\left(N(v) \cup N\left(w_{i}^{+}\right)\right)$for each $w_{i} \in Q$. It follows from (11), (12) and (4) that $p=2$. So, $k=1, p=2$ and $w_{2}^{-} w_{2}^{+} \in E(G)$.

If $w_{1} \neq x$ then we can obtain $w_{1}^{-} w_{1}^{+} \in E(G)$ by considering a $y-x$ path $\overleftarrow{P}_{n}^{\Delta}$ and using the above arguments.

Case 2. $k=p$.

We assume that $x=w_{1}$. (Otherwise we can consider a $y-x$ path $\overleftarrow{P}_{n}^{\Delta}$ and use the same arguments as in Case 1). Set $Q=\left\{w_{1}, \ldots, w_{p-1}\right\}$. Let $a_{1} a_{2} a_{3} a_{1}$ be a triangle with $w_{p} \in\left\{a_{1}, a_{2}\right\}$. By Property 4 all of (4), (5) and (6) hold because $Q \cap\left\{a_{1}, a_{2}\right\}=\varnothing$. Furthermore, since the vertices $w_{p}$ and $w_{i}^{+}$can be adjacent for each $w_{i} \in Q$, (9) also holds. On the other hand the inequality (12) also holds, because $v \notin Q^{+}$and $v \in N\left(w_{i}\right) \backslash\left(N(v) \cup N\left(w_{i}^{+}\right)\right)$for each $w_{i} \in Q$. It follows from (9), (12) and (4) that

$$
\sum_{w_{i} \in Q}\left|N(v) \cap N\left(w_{i}^{+}\right)\right|=\varepsilon\left(Q, Q^{+}\right)+p-1
$$

This implies that $w_{i}^{+} w_{p} \in E(G)$ for $i=1, \ldots, p-1$. Now we have that $p \geq\left|N(v) \cap N\left(w_{i}^{+}\right)\right| \geq\left|N\left(w_{p}\right) \backslash\left(N(v) \cup N\left(w_{i}^{+}\right)\right)\right|$and $\left\{v, w_{1}^{+}, \ldots, w_{p-1}^{+}\right\} \subseteq N\left(w_{p}\right) \backslash$ $\left(N(v) \cup N\left(w_{i}^{+}\right)\right)$. Therefore, for each $i=1, \ldots, p-1$ we have

$$
N\left(w_{p}\right) \backslash\left(N(v) \cup N\left(w_{i}^{+}\right)\right)=\left\{v, w_{1}^{+}, \ldots, w_{p-1}^{+}\right\}
$$

Since $w_{p}^{+} \in N\left(w_{p}\right) \backslash N(v),(14)$ implies that

$$
w_{i}^{+} w_{p}^{+} \in E(G) \quad \text { for each } i=1, \ldots, p-1
$$

Suppose that $w_{p}^{-} w_{p}^{+} \notin E(G)$. Then, by the definition of $p, w_{p} w_{p}^{++} \in E(G)$. Since $w_{p}^{++} \in N\left(w_{p}\right) \backslash N(v)$, (14) implies that $w_{1}^{+} w_{p}^{++} \in E(G)$. By (15), we also have that $w_{1}^{+} w_{p}^{+} \in E(G)$. Then $G$ has an $x-y$ path $P_{n+1}^{\Delta_{1}}=x v w_{p} \vec{P}_{n}^{A} w_{1}^{+} w_{p}^{+} \vec{P}_{n}^{A} y$ with $\Delta_{1}=$ $w_{1}^{+} w_{p}^{+} w_{p}^{++} w_{1}^{+}$; a contradiction. So, $w_{p}^{-} w_{p}^{+} \in E(G)$ and, therefore, $w_{p-1}^{+} \neq w_{p}^{-}$. Moreover, (14) implies that $w_{1}^{+} w_{p}^{-} \in E(G)$ since $w_{p}^{-} \in N\left(w_{p}\right) \backslash N(v)$.

If $p \geq 3$ then $G$ has an $x-y$ path $P_{n+1}^{A_{1}}=x v w_{p-1} \overleftarrow{P}_{n}^{\Delta} w_{1}^{+} w_{p}^{-} \overleftarrow{P}_{n}^{A} w_{p-1}^{+} w_{p} \vec{P}_{n}^{\Delta} y$ with $\Delta_{1}=w_{p-1}^{+} w_{p} w_{p}^{+} w_{p-1}^{+} ;$a contradiction. Therefore, $p=2$ and $w_{2}^{-} w_{2}^{+} \in E(G)$.

Now using the properties above we will obtain a contradiction. Let $v \in U_{2}$, $W_{v}=\left\{w_{1}, w_{2}\right\}$ and assume that $w_{1}$ occur on $\vec{P}_{n}^{A}$ before $w_{2}$. W.l.o.g. we may assume that $v y \notin E(G)$. Then, by Property $6, w_{2}^{-} w_{2}^{+} \in E(G)$. Clearly, $w_{1}^{+} w_{2} \in E(G)$ because $\left|N(v) \cap N\left(w_{1}^{+}\right)\right| \geq 2, N(v) \cap N\left(w_{1}^{+}\right) \subseteq W_{v}$ and $\left|W_{v}\right|=2$. Since $G$ is 2-connected and $n \leq|V(G)|-2$ we have that $N\left(P_{n}^{\Delta}\right) \backslash\{v\} \neq \varnothing$. Let us show

$$
u w_{2}^{-} \notin E(G) \quad \text { for each } u \in N\left(P_{n}^{\Delta}\right) \backslash\{v\}
$$

Suppose $u w_{2}^{-} \in E(G)$ for some $u \in N\left(P_{n}^{A}\right) \backslash\{v\}$. Then, by Property $2, u \in U_{2}$. For the vertex $g=w_{2}^{-}$we have $g^{-} \neq w_{1}$ and $g \in W_{u} \backslash\{x, y\}$. Hence, by Property 6, $g^{-} g^{+} \in E(G)$. But then for the triangle $g^{-} g g^{+} g^{-}$we have $\left\{g^{-}, g\right\} \cap W_{v}=\varnothing$. This contradicts Property 5. So, (16) is proved. 
Now let us show that

$$
u w_{2} \notin E(G) \quad \text { for each } u \in N\left(P_{n}^{S}\right) \backslash\{v\}
$$

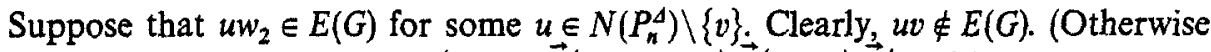
there exists an $x-y$ path $P_{n+2}^{\Delta_{1}}=x \vec{P}_{n}^{\Delta} w_{1} v u w_{2} w_{1}^{+} \vec{P}_{n}^{\Delta} w_{2}^{-} w_{2}^{+} \vec{P}_{n}^{\Delta} y$ with $\Delta_{1}=v u w_{2} v$, a contradiction.)

We have $w_{2}^{-}, v, u \in N\left(w_{2}\right) \backslash\left(N\left(w_{2}^{-}\right) \cup N(v)\right)$ and $d\left(w_{2}^{-}, v\right)=2$. Therefore, by Proposition $1,\left|N\left(w_{2}^{-}\right) \cap N(v)\right| \geq 3$. Since $\left|W_{v}\right|=2$ there is a vertex $v_{1} \in\left(N(v) \cap N\left(w_{2}^{-}\right)\right) \backslash W_{v}$ which contradicts (16). So (17) is proved.

Consider a vertex $u \in N\left(P_{n}^{d}\right) \backslash\{v\}$. By (17) and Property 2 we have $u \in U_{2}$. Using Property 5 with the triangle $w_{2}^{-} w_{2} w_{2}^{+} w_{2}$, the vertex $u$ and the path $\vec{P}_{n}^{\Delta}$ we obtain that $u y \in E(G)$. Then $u x \notin E(G)$ because $|N(u) \cap\{x, y\}| \leq 1$. Let $W_{u}=\left\{g_{1}, g_{2}\right\}$ where $g_{2}=y$. Then, by Property $6, g_{1}^{-} g_{1}^{+} \in E(G)$.

Using Property 5 with the triangle $w_{2}^{+} w_{2} w_{2}^{-} w_{2}^{+}$, the vertex $u$ and the path $\overleftarrow{P}_{n}^{4}$, we obtain $u w_{2}^{+} \in E(G)$. Suppose that $w_{2}^{+} \neq y$. Then $g_{1}=w_{2}^{+}$and $g_{1}^{-} g_{1}^{+} \in E(G)$. This implies $w_{2}^{-} g_{1}^{+} \in E(G)$. (Otherwise $w_{2}^{-}, g_{1}^{+}, u \in N\left(g_{1}\right) \backslash\left(N(u) \cup N\left(g_{1}^{+}\right)\right)$and, by Proposition $1,\left|N(u) \cap N\left(g_{1}^{+}\right)\right| \geq 3$. Since $\left|W_{u}\right|=2$ there exists a vertex $u_{1} \in\left(N(u) \cap N\left(g_{1}^{+}\right)\right) \backslash$ $W_{u}$ and an $x-y$ path $P_{n+2}^{A_{1}}=x \vec{P}_{n}^{\Delta} g_{1} u u_{1} g_{1}^{+} \vec{P}_{n}^{A} y$ with $A_{1}=w_{2}^{-} w_{2} w_{2}^{+} w_{2}^{-}$, a contradiction.) But now we obtain an $x-y$ path $P_{n+1}^{\Delta_{1}}=x \vec{P}_{n}^{\Delta} w_{1} v w_{2} w_{1}^{+} \vec{P}_{n}^{\Delta} w_{2}^{-} g_{1} g_{1}^{+} \vec{P}_{n}^{\Delta} y$ with $\Delta_{1}=w_{2}^{-} g_{1} g_{1}^{+} w_{2}^{-}$, a contradiction.

So, $w_{2}^{+}=g_{2}=y$. Then using Property 5 with the triangle $g_{1}^{-} g_{1} g_{1}^{+} g_{1}^{-}$, the vertex $v$ and the $x-y$ path $\vec{P}_{n}^{d}$, we obtain $w_{1} \in\left\{g_{1}^{-}, g_{1}\right\}$. If $g_{1}=w_{1}$ then $N(v) \cap N\left(w_{1}^{+}\right)=$ $\left\{w_{1}, w_{2}\right\}, \quad\left\{u, v, w_{1}^{+}\right\} \subseteq N\left(w_{1}\right) \backslash N\left(w_{1}^{+}\right)$and $v, w_{1}^{+} \in N\left(w_{1}\right) \backslash\left(N(v) \cap N\left(w_{1}^{+}\right)\right)$imply $u v \in E(G)$. But then there is a path $P_{n+1}^{A_{2}}=x \vec{P}_{n}^{\Delta} w_{1} u v w_{2} w_{1}^{+} \vec{P}_{n}^{\Delta} w_{2}^{-} y$ with $\Delta_{2}=w_{1} u v w_{1}$, a contradiction. So, $w_{1}=g_{1}^{-}$.

Now we have: $w_{1}^{+}=g_{1}, w_{2}^{+}=y=g_{2}, g_{1}^{-} g_{1}^{+} \in E(G), w_{2}^{-} w_{2}^{+} \in E(G), w_{1}^{+} w_{2} \in E(G)$, $d\left(w_{1}, u\right)=2$ and $w_{1}^{+} \in N\left(w_{1}\right) \cap N(u)$. Clearly, $N(u) \cap N\left(w_{1}\right) \subseteq W_{u}$. (Otherwise there exists a vertex $u_{1} \in\left(N(u) \cap N\left(w_{1}\right)\right) \backslash W_{u}$ and an $x-y$ path $\vec{P}_{n+2}^{\Delta_{1}}=x \vec{P}_{n}^{\Delta} w_{1} u_{1} u w_{1}^{+} \vec{P}_{n}^{\Delta} y$ with $\Delta_{1}=w_{2}^{-} w_{2} w_{2}^{+} w_{2}^{-}$, a contradiction.) Hence, using Proposition 1, we obtain

$$
2 \geq\left|N\left(w_{1}\right) \cap N(u)\right| \geq\left|N\left(w_{1}^{+}\right) \backslash\left(N\left(w_{1}\right) \cup N(u)\right)\right|
$$

On the other hand, we have $w_{1} u, u w_{2} \notin E(G)$ and $u, w_{1}, w_{2} \in N\left(w_{1}^{+}\right)$. Then $w_{1} w_{2} \in$ $E(G)$. (Otherwise $u, w_{1}, w_{2} \in N\left(w_{1}^{+}\right) \backslash\left(N(u) \cup N\left(w_{1}\right)\right)$ which contradicts (18).) Now we obtain an $x-y$ path $P_{n+1}^{\Delta_{1}}=x \vec{P}_{n}^{\Delta} w_{1} v w_{2} w_{1}^{+} \vec{P}_{n}^{\Delta} w_{2}^{-} y$ with $\Delta_{1}=w_{1} v w_{2} w_{1}$, a contradiction. The proof of the lemma is complete.

Acknowledgement. We thank R. Häggkvist for discussions and many helpful comments. We also thank the referees for their remarks and suggestions which led to this improved version.

\section{References}

1. Asratian, A.S.: A criterion for some hamiltonian graphs to be Hamilton-connected. Australasian J. Comb. 10, 193-198 (1994)

2. Asratian, A.S., Broersma, H.J., van den Heuvel, J., Veldman, H.J.: On graphs satisfying a local Ore-type condition. J. Graph Theory 21, 1-10 (1996) 
3. Asratian, A.S., Khachatrian, N.K.: Investigation of graph's hamiltonicity using neighborhoods of vertices (Russian). Doclady Acad. Nauk Armenian SSR 81, 103-106 (1985)

4. Asratian, A.S., Sarkisian, G.V.: On cyclic properties of some hamiltonian graphs (Russian). Diskretnaja Matematika 3, 91-104 (1991)

5. Bondy, J.A.: Pancyclic graphs 1. J. Comb. Theory Ser. B 11, 80-84 (1971)

6. Bondy, J.A., Murty, U.S.R.: Graph Theory with Applications, MacMillan, London and Elsevier, New York

7. Xiaota, Cai: On the panconnectivity of Ore graph. Scientia Sinica, 27, 684-694 (1984)

8. Häggkvist, R., Faudree, R.J., Schelp, R.H.: Pancyclic graphs-connected Ramsey number. Ars Combinatoria 11, 37-49 (1981)

9. Hasratian, A.S., Khachatrian, N.K.: Some localization theorems on hamiltonian circuits, J. Comb. Theory Ser. B 49, 287-294 (1990)

10. Ore, O.: Note on hamiltonian circuits. Amer. Math. Monthly 6755 (1960)

11. Zhang Ke Min, Holton, D.A., Bau Sheng: On vertex-pancyclic graphs. Preprint (1989) 